\title{
E-Bayesian Estimation for the Exponential Model Based on Record Statistics
}

\author{
Hassan M. Okasha* \\ Department of Statistics, King Abdul Aziz University, P.O.Box: 80203, Jeddah 21589, Saudi Arabia
}

\section{ARTICLE INFO}

Article History

Received 23 Mar 2017

Accepted 18 Mar 2019

\section{Keywords}

E-Bayes estimation

Bayes estimation

Exponential distribution

Upper record statistics values

Squared error loss function

Monte Carlo simulation

2000 Mathematics Subject

Classification: 62F15; 62F30;

62F86

\section{ABSTRACT}

This paper is concerned with using the E-Bayesian method for computing estimates for the parameter and reliability function of the Exponential distribution based on a set of upper record statistics values. The estimates are derived based on a conjugate prior for the scale parameter and squared error loss function. A comparisons between the new method and the corresponding Bayes technique are made using the Monte Carlo simulation.

(c) 2019 The Authors. Published by Atlantis Press SARL. This is an open access article distributed under the CC BY-NC 4.0 license (http://creativecommons.org/licenses/by-nc/4.0/).

\section{INTRODUCTION}

Let $X_{1}, X_{2}, \cdots$ be a sequence of identically independent distribution (iid) random variables with probability density function (pdf) $f(x)$. For $n \geq 1$, define

$$
U_{1}=1, \quad U_{n+1}=\min \left\{j: j>U_{n}, X_{j}>X_{U_{n}}\right\}
$$

The sequence $\left\{X_{U_{n}}\right\}\left(\left\{U_{n}\right\}\right)$ is known as upper record statistics (record times). These statistics are of interest and important in several reallife problems involving weather, economics and sports data. The statistical study of record values started with Chandler [1] has now spread in different directions. For more details and applications in the record values, see Ahsanullah [2] and Arnold et al. [3].

Consider the one-parameter exponential $(\operatorname{Exp}(\theta))$ distribution with pdf

$$
f(x)=\theta e^{-\theta x}, \quad x>0, \quad \theta>0,
$$

and the reliability function

$$
R(t)=e^{-\theta t}, \quad t>0
$$

The exponential distribution plays an important role in life testing problems. A great deal of research has been done on estimating the parameters of the exponential distribution using both classical and Bayesian techniques, and a very good summary of this work can be found in Johnson et al. [4]. There are also some papers on estimation and prediction for exponential parameters based on record and censored samples. See, for example, Balasubramanian and Balakrishnan [5], Chandrasekar et al. [6], and Ahmadi et al. [7] and references therein. Soliman [8] obtained the Bayes estimates using the symmetric (squared error) loss function.

*Email: hokasha45@gmail.com

Home address: Department of Mathematics, Faculty of Science, Al-Azhar University, Nasr City, 11884, Cairo, Egypt; E-mail: hassanokasha@yahoo.com. 


\section{BAYESIAN ESTIMATION}

Suppose we observe $n$ upper record values $X_{U_{1}}=x_{1}, X_{U_{2}}=x_{2}, \ldots, X_{U_{n}}=x_{n}$ from $\operatorname{Exp}(\theta)$ distribution with pdf given by (1). The likelihood function (LF) can be written as

$$
L(\theta \mid \underline{\mathbf{x}})=\prod_{i=1}^{n-1} h\left(x_{i}\right) f\left(x_{n}\right)
$$

where $\underline{\mathrm{x}}=\left(x_{1}, x_{2}, \ldots, x_{n}\right)$ and $h($.$) is the hazard function corresponding to the \operatorname{pdf} f($.$) . It follows, from (1), (2) and (3), that$

$$
L(\theta \mid \underline{\mathrm{x}})=\theta^{n} e^{-x_{n} \theta} .
$$

We use the following gamma conjugate prior density for the parameter $\theta$

$$
g(\theta \mid \alpha, \beta)=\frac{\beta^{\alpha}}{\Gamma(\alpha)} \theta^{\alpha-1} e^{-\beta \theta}, \quad \theta>0,
$$

where $\alpha>0$ and $\beta>0$. This prior was first used by Papadopoulos [9]. The posterior density of $\theta$ given $\underline{x}$ can be obtained from (4) and (5) and written as

$$
q(\theta \mid \underline{\mathrm{x}})=\kappa \theta^{n+\alpha-1} e^{-\left(\beta+x_{n}\right) \theta}, \theta>0
$$

where

$$
\kappa=\frac{\left(\beta+x_{n}\right)^{n+\alpha}}{\Gamma(n+\alpha)}
$$

Under the squared error loss function, the Bayes estimate of $\theta$ can be shown to be

$$
\hat{\theta}_{B S}(\alpha, \beta)=\frac{n+\alpha}{\beta+x_{n}}
$$

For more details about the squared error loss function, see, for example, Soliman [8].

The Bayes estimate of the reliability, $R_{B S}$, based on the squared error loss function is obtained from (2) and (6) as

$$
\hat{R}_{B S}(t)=\left(\frac{\beta+x_{n}}{\beta+x_{n}+t}\right)^{n+\alpha} .
$$

\section{E-BAYESIAN ESTIMATION}

According to Han [10], the prior parameters $\alpha$ and $\beta$ should be selected to guarantee that $g(\theta \mid \alpha, \beta)$ is a decreasing function of $\theta$. The derivative of $g(\theta \mid \alpha, \beta)$ with respect to $\theta$ is

$$
\frac{d g(\theta \mid \alpha, \beta)}{d \theta}=\frac{\beta^{\alpha}}{\Gamma(\alpha)} \theta^{\alpha-2} e^{-\beta \theta}[(\alpha-1)-\beta \theta]
$$

Note that $\alpha>0, \beta>0$, and $\theta>0$, it follows $0<\alpha<1, \beta>0$ due to $\frac{d g(\theta \mid \alpha, \beta)}{\theta}<0$, and therefore $g(\theta \mid \alpha, \beta)$ is a decreasing function of $\theta$. Assuming that $\alpha$ and $\beta$ are independent with bivariate density function

$$
\pi(\alpha, \beta)=\pi_{1}(\alpha) \pi_{2}(\beta),
$$

then, the E-Bayesian estimate of $\theta$ (expectation of the Bayesian estimate of $\theta$ ) can be written as

$$
\hat{\theta}_{E B}=E(\theta \mid \underline{X})=\iint_{\rho} \hat{\theta}_{B S}(\alpha, \beta) \pi(\alpha, \beta) d \alpha d \beta,
$$

where $\rho$ is the domain of $\alpha$ and $\beta, \hat{\theta}_{B}(\alpha, \beta)$ is the Bayes estimate of $\theta$ given by (8). For more details, see [11-15]. 


\subsection{E-Bayesian Estimation for $\theta$}

E-Bayesian estimation of $\theta$ is obtained based on three different distributions of the hyperparameters $\alpha$ and $\beta$. These distributions are used to investigate the influence of the different prior distributions on the E-Bayesian estimation of $\theta$.

The following distributions of $\alpha$ and $\beta$ may be used

$$
\begin{aligned}
& \pi_{1}(\alpha, \beta)=\frac{1}{s B(u, v)} \alpha^{u-1}(1-\alpha)^{v-1}, \quad 0<\alpha<1, \quad 0<\beta<s, \\
& \left.\pi_{2}(\alpha, \beta)=\frac{2}{s^{2} B(u, v)}(s-\beta) \alpha^{u-1}(1-\alpha)^{v-1}, \quad 0<\alpha<1, \quad 0<\beta<s,\right\} \text {. } \\
& \pi_{3}(\alpha, \beta)=\frac{2 \beta}{s^{2} B(u, v)} \alpha^{u-1}(1-\alpha)^{v-1}, \quad 0<\alpha<1, \quad 0<\beta<s,
\end{aligned}
$$

where $B(u, v)$ is the beta function. For $\pi_{1}(\alpha, \beta)$, the E-Bayesian estimate of $\theta$ is obtained from (8) and (12) as

$$
\begin{aligned}
\hat{\theta}_{E B S 1} & =\iint_{D} \hat{\theta}_{B S}(\alpha, \beta) \pi_{1}(\alpha, \beta) d \beta d \alpha, \\
& =\frac{1}{s B(u, v)} \int_{0}^{1} \int_{0}^{s}\left(\frac{n+\alpha}{\beta+x_{n}}\right) \alpha^{u-1}(1-\alpha)^{v-1} d \beta d \alpha . \\
& =\frac{1}{s}\left(n+\frac{u}{u+v}\right) \ln \left(\frac{s+x_{n}}{x_{n}}\right) .
\end{aligned}
$$

Similarly, the E-Bayesian estimates of $\theta$ based on $\pi_{2}(\alpha, \beta)$ and $\pi_{3}(\alpha, \beta)$ are computed and given, respectively, by

$$
\hat{\theta}_{E B S 2}=\frac{2}{s}\left(n+\frac{u}{u+v}\right)\left[\frac{s+x_{n}}{s} \ln \left(\frac{s+x_{n}}{x_{n}}\right)-1\right],
$$

and

$$
\hat{\theta}_{E B S 3}=\frac{2}{s}\left(n+\frac{u}{u+v}\right)\left[1-\frac{x_{n}}{s} \ln \left(\frac{s+x_{n}}{x_{n}}\right)\right] .
$$

\subsection{E-Bayesian Estimation for the Reliability}

Based on the squared error loss function, the E-Bayesian estimates of the reliability function is computed for the three different distributions of the hyperparameters $\alpha$ and $\beta$ given by (12). For $\pi_{1}(a, b)$, the E-Bayesian estimate of the reliability is obtained from (9), (11) and (12) as

$$
\begin{aligned}
\hat{R}_{E B S 1} & =\iint_{D} \hat{R}_{B S}(t) \pi_{1}(a, b) d b d a \\
& =\frac{1}{s B(u, v)} \int_{0}^{1} \int_{0}^{s}\left(\frac{\beta+x_{n}}{\beta+x_{n}+t}\right)^{n+\alpha} \alpha^{u-1}(1-\alpha)^{v-1} d \beta d \alpha . \\
& =\frac{1}{s B(u, v)} \int_{0}^{s}\left(\frac{\beta+x_{n}}{\beta+x_{n}+t}\right)^{n}\left(\int_{0}^{1} e^{\alpha \ln \left(\frac{\beta+x_{n}}{\beta+x_{n}+t}\right)} \alpha^{u-1}(1-\alpha)^{v-1} d \alpha\right) d \beta . \\
& =\frac{1}{s} \int_{0}^{s}\left(\frac{\beta+x_{n}}{\beta+x_{n}+t}\right)^{n} F_{1: 1}\left(u ; u+v ; \ln \left(\frac{\beta+x_{n}}{\beta+x_{n}+t}\right)\right) d \beta,
\end{aligned}
$$

where, $F_{1: 1}$ (.,.;.) is the generalized hypergeometric function [see Gradshteyn and Ryzhik [16] (formula 3.383 (1))]. Similarly, the E-Bayesian estimates of the reliability based on $\pi_{2}(a, b)$ and $\pi_{3}(a, b)$ are computed and given, respectively, by

$$
\hat{R}_{E B S 2}=\frac{2}{s^{2}} \int_{0}^{s}(s-\beta)\left(\frac{\beta+x_{n}}{\beta+x_{n}+t}\right)^{n} F_{1: 1}\left(u ; u+v ; \ln \left(\frac{\beta+x_{n}}{\beta+x_{n}+t}\right)\right) d \beta,
$$

and

$$
\hat{R}_{E B S 3}=\frac{2}{s^{2}} \int_{0}^{s} \beta\left(\frac{\beta+x_{n}}{\beta+x_{n}+t}\right)^{n} F_{1: 1}\left(u ; u+v ; \ln \left(\frac{\beta+x_{n}}{\beta+x_{n}+t}\right)\right) d \beta .
$$


The integrals in (16), (17) and (18) can not be computed analytically in simple closed forms and numerical computations must be used for computing the E-Bayesian estimates of the reliability functions based on squared error loss function. The Maple ${ }^{T M} 12$ is used for the numerical computations.

\section{PROPERTIES OF E-BAYESIAN ESTIMATION}

Now we discuss the relations among $\hat{\theta}_{E B S i}(i=1,2,3)$ and the relations between $\hat{R}_{E B S i}(i=1,2,3)$.

1. Relations among $\hat{\theta}_{E B S i}(i=1,2,3)$

Lemma 4.1. Let $0<s<x_{n}$ and $\hat{\theta}_{E B S i}(i=1,2,3)$ be given by (13), (14) and (15) then

i. $\hat{\theta}_{E B S 2}<\hat{\theta}_{E B S 1}<\hat{\theta}_{E B S 3}$.

ii. $\lim _{x_{n} \rightarrow \infty} \hat{\theta}_{E B S 1}=\lim _{x_{n} \rightarrow \infty} \hat{\theta}_{E B S 2}=\lim _{x_{n} \rightarrow \infty} \hat{\theta}_{E B S 3}$.

Proof. See Appendix A.

2. Relations among $\hat{R}_{E B S i}(i=1,2,3)$.

Lemma 4.2. Let $0<s<x_{n}$ and $\hat{\theta}_{E B S i}(i=1,2,3)$ be given by (16), (17) and (18) then

$$
\lim _{x_{n} \rightarrow \infty} \hat{R}_{E B S 1}=\lim _{x_{n} \rightarrow \infty} \hat{R}_{E B S 2}=\lim _{x_{n} \rightarrow \infty} \hat{R}_{E B S 3}
$$

Proof. See Appendix A.

Remark. From (16), (17) and (18), we have

$$
\begin{aligned}
\hat{R}_{E B S 3}-\hat{R}_{E B S 1} & =\hat{R}_{E B S 1}-\hat{R}_{E B S 2} \\
& =\frac{1}{s^{2}}\left\{\int_{0}^{s}(2 \beta-s)\left(\frac{\beta+x_{n}}{\beta+x_{n}+t}\right)^{n} F_{1: 1}\left(u ; u+v ; \ln \left(\frac{\beta+x_{n}}{\beta+x_{n}+t}\right)\right) d \beta\right\}
\end{aligned}
$$

we note that, this integral may not be computed analytically, therefore, we have solved it numerically using Maple ${ }^{T M} 12$. The numerical results show that this integral usually positive. It follows that

$$
\hat{R}_{E B S 2}<\hat{R}_{E B S 1}<\hat{R}_{E B S 3}
$$

\section{MONTE-CARLO SIMULATION AND COMPARISONS}

In this section, a Monte Carlo simulation is used for a comparison of the Bayes and E-Bayes techniques of estimation. The following steps are considered:

- For given values of the prior parameters $(u, v)$ and $(0, s)$ we generate $\alpha$ and $\beta$ from the beta and uniform priors (12), respectively.

- For given values of $(\alpha, \beta)$ we generate $\theta$ from the gamma prior density (5).

- For known values of $\theta$, an upper record sample of size $n$ is then generated from the density of the $\operatorname{Exp}(\theta)$ distribution defined by (1) using the transformation: $X_{i}=F^{-1}\left(U_{i}\right)=-\frac{1}{\theta} \ln \left(1-U_{i}\right)$ where $U_{i}$ is the uniformly distributed random variate. The sequence of record values was generated as follows: (i) Generate the 1 st value and record it as the first record value. (ii) Generate the 2 nd value, if it is greater than the previous, then record it as the 2 nd record value, if not generate another value and so on. The codes of Maple ${ }^{12}$ are used to generate from the gamma, beta and uniform distributions.

- Based on the squared loss function, the estimates $\hat{\theta}_{B S}, \hat{\theta}_{E B S 1}, \hat{\theta}_{E B S 2}$ and $\hat{\theta}_{E B S 3}$ of $\theta$ are computed from (8), (13), (14) and (15).

- Based on the squared loss function, the estimates $\hat{R}_{B S}, \hat{R}_{E B S 1}, \hat{R}_{E B S 2}$ and $\hat{R}_{E B S 3}$ of $R$ are computed from (9), (16), (17) and (18).

- The quantities $(\hat{\phi}-\phi)^{2}$ are computed where $\hat{\phi}$ stands for an estimate of $\phi$. 
- The above steps are repeated 1000 times and the estimated risks (ER) of the estimates are computed by averaging the squared deviations over 1000 repetitions:

$$
E R(\hat{R})=\frac{1}{1000} \sum(\hat{R}-R)^{2}
$$

- The computational results are displayed in Tables 1 and 2.

\section{CONCLUDING REMARKS}

In this paper, E-Bayes and Bayes methods are used for estimating the parameter and the reliability function of the exponential distribution based on record statistics. The Monte-Carlo simulation and comparisons are used for computing E-Bayes and Bayes estimates. We will present the conclusions in the following points:

a. Generally, the ER of the E-Bayes estimate of $\theta$ and $R$ are the smallest ERs. On the other hand, the ER of the E-Bayes estimates of $\theta$ and $R$ based on the squared loss function are less than the ER of their corresponding Bayes estimates.

b. It has been noticed, from Tables 1 and 2, that the E-Bayes estimates, in most cases, tend to be more efficient than the Bayes estimates in the sense of having smaller ERs of the estimates. Also, the ERs of the estimates increases as $n$ increases and the E-Bayes estimates have the smallest ERs as compared with their corresponding Bayes estimates. By increasing $n$, the computations in Tables 1 and 2 show that the E-Bayes estimates (based on squared error loss) are better than the Bayes in the sense of comparing the ERs of the estimates.

c. The author suggest take beta and uniform distribution as the priors of the hyperparameters $\alpha$ and $\beta$, respectively. The work in this paper showed that the E-Bayesian estimation method is both efficient and easy to perform.

Table 1 Estimated risks (ERs) of the estimates of $\hat{\theta}_{B S}, \hat{\theta}_{E B S 1}, \hat{\theta}_{E B S 2}$, and $\hat{\theta}_{E B S 3}$.

\begin{tabular}{|c|c|c|c|c|c|c|}
\hline$n$ & $s$ & $(u, v)$ & $\hat{\theta}_{B S}$ & $\hat{\theta}_{E B S 1}$ & $\hat{\theta}_{E B S 2}$ & $\hat{\theta}_{E B S 3}$ \\
\hline \multirow[t]{4}{*}{5} & \multirow[t]{2}{*}{0.1} & $(3,2)$ & 0.2017442307 & 0.1883297898 & 0.1886577740 & 0.1880020030 \\
\hline & & $(4,3)$ & 0.1929137491 & 0.1810729415 & 0.1813785003 & 0.1807675613 \\
\hline & \multirow[t]{2}{*}{0.2} & $(3,2)$ & 0.3982663752 & 0.3709431448 & 0.3734597677 & 0.3684320680 \\
\hline & & $(4,3)$ & 0.3809784612 & 0.3568132202 & 0.3591615226 & 0.3544699350 \\
\hline \multirow[t]{4}{*}{7} & \multirow[t]{2}{*}{0.1} & $(3,2)$ & 0.2184657662 & 0.2076281370 & 0.2078776591 & 0.2073786998 \\
\hline & & $(4,3)$ & 0.2095240406 & 0.1999569461 & 0.2001895177 & 0.1997244518 \\
\hline & \multirow[t]{2}{*}{0.2} & $(3,2)$ & 0.4329793602 & 0.4108522767 & 0.4127976468 & 0.4089093544 \\
\hline & & $(4,3)$ & 0.4153715457 & 0.3958026592 & 0.3976190127 & 0.3939885204 \\
\hline \multirow[t]{4}{*}{10} & \multirow[t]{2}{*}{0.1} & $(3,2)$ & 0.2444457070 & 0.2357117460 & 0.2358954264 & 0.2355280883 \\
\hline & & $(4,3)$ & 0.2350112326 & 0.2273010337 & 0.2274735737 & 0.2271285123 \\
\hline & \multirow[t]{2}{*}{0.2} & $(3,2)$ & 0.4859920654 & 0.4681456700 & 0.4695978012 & 0.4666940671 \\
\hline & & $(4,3)$ & 0.4673214397 & 0.4515405872 & 0.4528968777 & 0.4501847724 \\
\hline \multirow[t]{4}{*}{15} & \multirow[t]{2}{*}{0.1} & $(3,2)$ & 0.3038514648 & 0.2966034956 & 0.2967692871 & 0.2964377198 \\
\hline & & $(4,3)$ & 0.2927368776 & 0.2927368776 & 0.2927368776 & 0.2861821773 \\
\hline & \multirow[t]{2}{*}{0.2} & $(3,2)$ & 0.6051030307 & 0.5902370221 & 0.5915542680 & 0.5889200020 \\
\hline & & $(4,3)$ & 0.5830482568 & 0.5699001678 & 0.5711311623 & 0.5686693798 \\
\hline \multirow[t]{4}{*}{20} & \multirow[t]{2}{*}{0.1} & $(3,2)$ & 0.3721301397 & 0.3655446192 & 0.3657206211 & 0.3653686418 \\
\hline & & $(4,3)$ & 0.3589087371 & 0.3530940504 & 0.3532596655 & 0.3529284546 \\
\hline & \multirow[t]{2}{*}{0.2} & $(3,2)$ & 0.7415262048 & 0.7279499471 & 0.7293427421 & 0.7265573663 \\
\hline & & $(4,3)$ & 0.7152647777 & 0.7032529605 & 0.7045548355 & 0.7019512817 \\
\hline \multirow[t]{4}{*}{25} & \multirow[t]{2}{*}{0.1} & $(3,2)$ & 0.4355546074 & 0.4294372101 & 0.4296175092 & 0.4292569430 \\
\hline & & $(4,3)$ & 0.4203504551 & 0.4149486454 & 0.4151222386 & 0.4147750800 \\
\hline & \multirow[t]{2}{*}{0.2} & $(3,2)$ & 0.8682786579 & 0.8556138104 & 0.8570607075 & 0.8541670942 \\
\hline & & $(4,3)$ & 0.8380568582 & 0.8268480177 & 0.8282007455 & 0.8254954489 \\
\hline \multirow[t]{4}{*}{30} & \multirow[t]{2}{*}{0.1} & $(3,2)$ & 0.4860639584 & 0.4803990052 & 0.4805801273 & 0.4802179156 \\
\hline & & $(4,3)$ & 0.4692934979 & 0.4642909207 & 0.4644610439 & 0.4641208303 \\
\hline & \multirow[t]{2}{*}{0.2} & $(3,2)$ & 0.9693228105 & 0.9575592737 & 0.9589969053 & 0.9561217672 \\
\hline & & $(4,3)$ & 0.9359658770 & 0.9255525959 & 0.9268965622 & 0.9242087416 \\
\hline
\end{tabular}


Table 2 Estimated risks (ERs) of the estimates of $\hat{R}_{B S}, \hat{R}_{E B S 1}, \hat{R}_{E B S 2}$, and $\hat{R}_{E B S 3}$.

\begin{tabular}{|c|c|c|c|c|c|c|c|}
\hline$n$ & $s$ & $(u, v)$ & $t$ & $\hat{\boldsymbol{R}}_{B S}$ & $\hat{\boldsymbol{R}}_{E B S 1}$ & $\hat{\boldsymbol{R}}_{E B S 2}$ & $\hat{R}_{E B S 3}$ \\
\hline \multirow[t]{8}{*}{5} & \multirow[t]{2}{*}{0.1} & $(3,2)$ & \multirow[t]{4}{*}{1} & 0.1506261589 & 0.1416593141 & 0.1418198901 & 0.1414987537 \\
\hline & & $(4,3)$ & & 0.1454107429 & 0.1373973487 & 0.1375500379 & 0.1372446747 \\
\hline & \multirow[t]{2}{*}{0.2} & $(3,2)$ & & 0.2374808510 & 0.2240232094 & 0.2248199445 & 0.2232265374 \\
\hline & & $(4,3)$ & & 0.2307616910 & 0.2186234287 & 0.2193885079 & 0.2178584147 \\
\hline & \multirow[t]{2}{*}{0.1} & $(3,2)$ & \multirow[t]{4}{*}{2} & 0.2390433964 & 0.2258201086 & 0.2260214050 & 0.2256188162 \\
\hline & & $(4,3)$ & & 0.2322617402 & 0.2203489319 & 0.2205422289 & 0.2201556391 \\
\hline & \multirow[t]{2}{*}{0.2} & $(3,2)$ & & 0.3244785603 & 0.3080374905 & 0.3088472262 & 0.3072277709 \\
\hline & & $(4,3)$ & & 0.3184187738 & 0.3034123420 & 0.3042004630 & 0.3026242355 \\
\hline \multirow[t]{8}{*}{7} & \multirow[t]{2}{*}{0.1} & $(3,2)$ & \multirow[t]{4}{*}{1} & 0.1664355735 & 0.1591245623 & 0.1592575537 & 0.1589915789 \\
\hline & & $(4,3)$ & & 0.1610567686 & 0.1545229758 & 0.1546493432 & 0.1543966162 \\
\hline & \multirow[t]{2}{*}{0.2} & $(3,2)$ & & 0.2644564078 & 0.2535854752 & 0.2542579002 & 0.2529130856 \\
\hline & & $(4,3)$ & & 0.2575898017 & 0.2477781682 & 0.2484238705 & 0.2471325025 \\
\hline & \multirow[t]{2}{*}{0.1} & $(3,2)$ & \multirow[t]{4}{*}{2} & 0.2657684489 & 0.2551011427 & 0.2552705990 & 0.2549316887 \\
\hline & & $(4,3)$ & & 0.2588495258 & 0.2492335958 & 0.2493963142 & 0.2490708796 \\
\hline & \multirow[t]{2}{*}{0.2} & $(3,2)$ & & 0.3620170205 & 0.3491360308 & 0.3498112707 & 0.3484607975 \\
\hline & & $(4,3)$ & & 0.3561606991 & 0.3443832818 & 0.3450414239 & 0.3437251457 \\
\hline \multirow[t]{8}{*}{10} & \multirow[t]{2}{*}{0.1} & $(3,2)$ & \multirow[t]{4}{*}{1} & 0.1902154074 & 0.1842778547 & 0.1843886372 & 0.1841670756 \\
\hline & & $(4,3)$ & & 0.1844215968 & 0.1791139476 & 0.1792190713 & 0.1790088271 \\
\hline & 0.2 & $(3,2)$ & & 0.3038538360 & 0.2951848142 & 0.2957580609 & 0.2946115861 \\
\hline & & $(4,3)$ & & 0.2965808362 & 0.2887456885 & 0.2892961584 & 0.2881952377 \\
\hline & 0.1 & $(3,2)$ & 2 & 0.3049681217 & 0.2964763742 & 0.2966205606 & 0.2963321890 \\
\hline & & $(4,3)$ & & 0.2976508212 & 0.2899859234 & 0.2901243769 & 0.1790088271 \\
\hline & 0.2 & $(3,2)$ & & 0.4140555534 & 0.4043222408 & 0.4048844289 & 0.4037600547 \\
\hline & & $(4,3)$ & & 0.4083744874 & 0.3994449368 & 0.3999942346 & 0.2898474711 \\
\hline 15 & 0.1 & $(3,2)$ & 1 & 0.2340248721 & 0.2292959584 & 0.2293955774 & 0.2291963409 \\
\hline & & $(4,3)$ & & 0.2274269669 & 0.2231936912 & 0.2232883215 & 0.2230990623 \\
\hline & 0.2 & $(3,2)$ & & 0.2784629712 & 0.2744421332 & 0.2745402336 & 0.2743440339 \\
\hline & & $(4,3)$ & & 0.3614559775 & 0.3554753200 & 0.3559590777 & 0.3549915706 \\
\hline & 0.1 & $(3,2)$ & 2 & 0.2784629712 & 0.2744421332 & 0.2745402336 & 0.2743440339 \\
\hline & & $(4,3)$ & & 0.3623921863 & 0.3565648314 & 0.3566862977 & 0.3564433655 \\
\hline & 0.2 & $(3,2)$ & & 0.4899697646 & 0.4832990205 & 0.4837533364 & 0.4828447059 \\
\hline & & $(4,3)$ & & 0.4848545413 & 0.4786939831 & 0.4791404803 & 0.4782474871 \\
\hline 20 & 0.1 & $(3,2)$ & 1 & 0.2784629712 & 0.2744421332 & 0.2745402336 & 0.2743440339 \\
\hline & & $(4,3)$ & & 0.2711134801 & 0.2675066825 & 0.2676000935 & 0.2674132727 \\
\hline & 0.2 & $(3,2)$ & & 0.4296598122 & 0.4243555945 & 0.4248220065 & 0.4238891869 \\
\hline & & $(4,3)$ & & 0.4214785019 & 0.4166494905 & 0.4171002457 & 0.4161987398 \\
\hline & 0.1 & $(3,2)$ & 2 & 0.4305594645 & 0.4254058032 & 0.4255227963 & 0.4252888103 \\
\hline & & $(4,3)$ & & 0.4223482520 & 0.4176644479 & 0.4177775148 & 0.4175513810 \\
\hline & 0.2 & $(3,2)$ & & 0.5503792577 & 0.5455705727 & 0.5459492432 & 0.5451919045 \\
\hline & & $(4,3)$ & & 0.5461373796 & 0.5416660740 & 0.5420407322 & 0.5412914174 \\
\hline 25 & 0.1 & $(3,2)$ & 1 & 0.3178489459 & 0.3143266763 & 0.3144231573 & 0.3142301961 \\
\hline & & $(4,3)$ & & 0.3098722414 & 0.3067067059 & 0.3067987487 & 0.3066146639 \\
\hline & 0.2 & $(3,2)$ & & 0.4805653588 & 0.4761550800 & 0.4765908567 & 0.4757193053 \\
\hline & & $(4,3)$ & & 0.4722484669 & 0.4722484669 & 0.4722484669 & 0.4677955214 \\
\hline & 0.1 & $(3,2)$ & 2 & 0.4814044100 & 0.4771361598 & 0.4772453945 & 0.4770269251 \\
\hline & & $(4,3)$ & & 0.4730625065 & 0.4691695475 & 0.4692754827 & 0.4690636124 \\
\hline & 0.2 & $(3,2)$ & & 0.5965025061 & 0.5929072065 & 0.5932271235 & 0.5925872917 \\
\hline & & $(4,3)$ & & 0.5931944183 & 0.5898286416 & 0.5901472156 & 0.5895100697 \\
\hline 30 & 0.1 & $(3,2)$ & 1 & 0.3484771341 & 0.3453542827 & 0.3454470559 & 0.3452615102 \\
\hline & & $(4,3)$ & & 0.3400443226 & 0.3372336112 & 0.3373222305 & 0.3371449924 \\
\hline & 0.2 & $(3,2)$ & & 0.5188557510 & 0.5151076985 & 0.5155110074 & 0.5147043908 \\
\hline & & $(4,3)$ & & 0.5105234191 & 0.5070875801 & 0.5074797408 & 0.5066954206 \\
\hline & 0.1 & $(3,2)$ & 2 & 0.5196315183 & 0.5160155862 & 0.5161166345 & 0.5159145379 \\
\hline & & $(4,3)$ & & 0.5112781009 & 0.5079703795 & 0.5080686382 & 0.5078721208 \\
\hline & 0.2 & $(3,2)$ & & 0.6287009073 & 0.6259093266 & 0.6261819411 & 0.6256367142 \\
\hline & & $(4,3)$ & & 0.6261979056 & 0.6235691251 & 0.6238420644 & 0.6232961875 \\
\hline
\end{tabular}




\section{ACKNOWLEDGMENTS}

The authors would like to thank the editors and the referees for careful reading and for fruitful comments which greatly improved the paper.

\section{REFERENCES}

1. K.N. Chandler, J. Royal, Stat. Soc. Ser. B. 14 (1952), 220-228.

2. M. Ahsanullah, Record Statistics, Nova Science, New York, 1995.

3. B.C. Arnold, N. Balakrishnan, H.N. Nagaraja, Records, John Wiley \& Sons, New York, 1998.

4. N.L. Johnson, S. Kotz, N. Balakrishnan, Continuous Univariate Distributions, second ed., vol. 1, John Wiley \& Sons, New York, 1994.

5. K. Balasubramanian, N. Balakrishnan, Stat. Hefte. 33 (1992), 203-216.

6. B. Chandrasekar, T. Leo Alexander, N. Balakrishnan, Commun. Stat. Theory. Methods. 31 (2002), 1675-1686.

7. J. Ahmadi, M. Doostparast, A. Parsian, Commun. Stat. Theory Methods. 34 (2005), 795-805.

8. A.A. Soliman, IEEE. Trans. Reliab. 54 (2005), 34-42.

9. A.S. Papadopoulos, IEEE Trans. Reliab. R-27 (1978), 369-371.

10. M. Han, Appl. Math. Model. 33 (2009), 1915-1922.

11. M. Han, Appl. Math. Model. 35 (2011), 2419-2424.

12. Z.F. Jaheen, H.M. Okasha, Appl. Math. Model. 35 (2011), 4730-4737.

13. H.M. Okasha, J. Wang, Appl. Math. Model. 40 (2016), 658-670.

14. H.M. Okasha, J. Egypt. Math. Soc. 22 (2014), 489-495.

15. H.M.Okasha, J. Adv. Res. Sci. Comput. 44 (2012), 34-45.

16. I.S. Gradshteyn, I.M. Ryzhik, Tables of Integrals, Series and Products, corrected and enlarged ed., Prepared by A. Jeffrey, Academic Press, Inc, London, 2007, pp. 96-99. 


\section{APPENDIX A}

\section{Proof of Lemma 4.1.}

i. From (13), (14) and (15), we have

$$
\hat{\theta}_{E B S 2}-\hat{\theta}_{E B S 1}=\hat{\theta}_{E B S 1}-\hat{\theta}_{E B S 3}=\frac{1}{s}\left(n+\frac{u}{u+v}\right)\left(\frac{s+2 x_{n}}{s} \ln \left(\frac{x_{n}+s}{x_{n}}\right)-2\right)
$$

For $-1<x<1$, we have: $\ln (1+x)=x-\frac{x^{2}}{2}+\frac{x^{3}}{3}-\frac{x^{4}}{4}+\ldots=\sum_{k=1}^{\infty}(-1)^{k-1} \frac{x^{k}}{k}$. Let $x=\frac{s}{x_{n}}$, when $0<s<x_{n}, 0<\frac{s}{x_{n}}<1$, we get

$$
\begin{aligned}
& {\left[\frac{s+2 x_{n}}{s} \ln \left(\frac{x_{n}+s}{x_{n}}\right)-2\right] } \\
= & \frac{s+2 x_{n}}{s}\left(\left(\frac{s}{x_{n}}\right)-\frac{1}{2}\left(\frac{s}{x_{n}}\right)^{2}+\frac{1}{3}\left(\frac{s}{x_{n}}\right)^{3}-\frac{1}{4}\left(\frac{s}{x_{n}}\right)^{4}+\frac{1}{5}\left(\frac{s}{x_{n}}\right)^{5}-\ldots\right)-2 \\
= & \left(\left(\frac{s}{x_{n}}\right)-\frac{1}{2}\left(\frac{s}{x_{n}}\right)^{2}+\frac{1}{3}\left(\frac{s}{x_{n}}\right)^{3}-\frac{1}{4}\left(\frac{s}{x_{n}}\right)^{4}+\frac{1}{5}\left(\frac{s}{x_{n}}\right)^{5}-\ldots\right)-2 \\
& +\left(2-\left(\frac{s}{x_{n}}\right)+\frac{2}{3}\left(\frac{s}{x_{n}}\right)^{2}-\frac{2}{4}\left(\frac{s}{x_{n}}\right)^{3}+\frac{2}{5}\left(\frac{s}{x_{n}}\right)^{4}-\ldots\right) \\
= & \left(\frac{s^{2}}{6 x_{n}^{2}}-\frac{s^{3}}{6 x_{n}^{3}}\right)+\left(\frac{3 s^{4}}{6 x_{n}^{4}}-\frac{2 s^{5}}{15 x_{n}^{5}}\right)+\ldots \\
= & \frac{s^{2}}{6 x_{n}^{2}}\left(1-\frac{s}{x_{n}}\right)+\frac{s^{4}}{60 x_{n}^{4}}\left(9-\frac{8 s}{x_{n}}\right)+\ldots>0 .
\end{aligned}
$$

According to (A.1) and (A.2), we have

$$
\hat{\theta}_{E B S 2}-\hat{\theta}_{E B S 1}=\hat{\theta}_{E B S 1}-\hat{\theta}_{E B S 3}>0,
$$

that is

$$
\hat{\theta}_{E B S 3}<\hat{\theta}_{E B S 1}<\hat{\theta}_{E B S 2} .
$$

ii. From (A.1) and (A.2), we get

$$
\begin{aligned}
\lim _{x_{n} \rightarrow \infty}\left(\hat{\theta}_{E B S 2}-\hat{\theta}_{E B S 1}\right) & =\lim _{x_{n} \rightarrow \infty}\left(\hat{\theta}_{E B S 1}-\hat{\theta}_{E B S 3}\right) \\
& =\frac{1}{s}\left(n+\frac{u}{u+v}\right) \lim _{x_{n} \rightarrow \infty}\left(\frac{s^{2}}{6 x_{n}^{2}}\left(1-\frac{s}{x_{n}}\right)+\frac{s^{4}}{60 x_{n}^{4}}\left(9-\frac{8 s}{x_{n}}\right)+\ldots\right) \\
& =0 .
\end{aligned}
$$

That is, $\lim _{x_{n} \rightarrow \infty} \hat{\theta}_{E B S 1}=\lim _{x_{n} \rightarrow \infty} \hat{\theta}_{E B S 2}=\lim _{x_{n} \rightarrow \infty} \hat{\theta}_{E B S 3}$.

Thus, the proof is complete.

\section{Proof of Lemma 4.2.}

i. From (19), we have

$$
\begin{aligned}
\lim _{x_{n} \rightarrow \infty}\left(\hat{R}_{E B S 3}-\hat{R}_{E B S 1}\right) & =\lim _{x_{n} \rightarrow \infty}\left(\hat{R}_{E B S 1}-\hat{R}_{E B S 2}\right) \\
& =\lim _{x_{n} \rightarrow \infty}\left\{\frac{1}{s^{2}} \int_{0}^{s}(2 \beta-s)\left(\frac{\beta+x_{n}}{\beta+x_{n}+t}\right)^{n} F_{1: 1}\left(u ; u+v ; \ln \left(\frac{\beta+x_{n}}{\beta+x_{n}+t}\right)\right) d \beta\right\} \\
& =0 .
\end{aligned}
$$

That is, $\lim _{x_{n} \rightarrow \infty} \hat{R}_{E B S 1}=\lim _{x_{n} \rightarrow \infty} \hat{R}_{E B S 2}=\lim _{x_{n} \rightarrow \infty} \hat{R}_{E B S 3}$.

Thus, the proof is complete. 